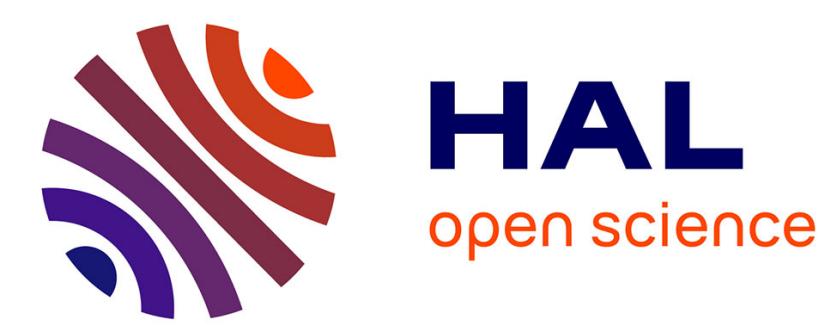

\title{
Construction and test results of the ATLAS EM barrel calorimeter and presampler
}

\author{
J.Y. Hostachy
}

\section{To cite this version:}

J.Y. Hostachy. Construction and test results of the ATLAS EM barrel calorimeter and presampler. Topical Seminar on Innovative Particle and Radiation Detectors 8, Oct 2002, Siena, Italy. pp.112-116. in2p3-00013681

\section{HAL Id: in2p3-00013681 https://hal.in2p3.fr/in2p3-00013681}

Submitted on 8 Sep 2003

HAL is a multi-disciplinary open access archive for the deposit and dissemination of scientific research documents, whether they are published or not. The documents may come from teaching and research institutions in France or abroad, or from public or private research centers.
L'archive ouverte pluridisciplinaire HAL, est destinée au dépôt et à la diffusion de documents scientifiques de niveau recherche, publiés ou non, émanant des établissements d'enseignement et de recherche français ou étrangers, des laboratoires publics ou privés. 


\title{
Construction and test results of the ATLAS EM barrel calorimeter and presampler
}

\author{
J.Y. Hostachy ${ }^{\mathrm{a}}$ on behalf of the ATLAS Liquid Argon Community \\ ${ }^{a}$ Institut des Sciences Nucléaires, Université Joseph Fourier, IN2P3-CNRS, \\ F-38026 Grenoble, France
}

The construction of the ATLAS liquid argon electromagnetic (EM) barrel calorimeter and presampler is well under way: modules and sectors for more than half a barrel have now been produced. Particular emphasis will be put on the qualification tests allowing this construction. The system: calorimeter module +2 presampler sectors has been exposed several times to muon, electron and photon beams at CERN. Results concerning muons and photons are shown. Energy resolution and the uniformity studies performed with electrons are presented in the same proceedings by Dr. M. Fanti.

\section{INTRODUTION}

The barrel electromagnetic calorimeter of the ATLAS experiment at CERN Large Hadron Collider (LHC) will be of the accordion liquid argon (LAr) type, a dectector concept developed by the RD3 collaboration [1] for use at LHC. The liquid argon calorimetry has been chosen because of its intrinsic linear behavior, response stability and radiation tolerance. To achieve a sufficient

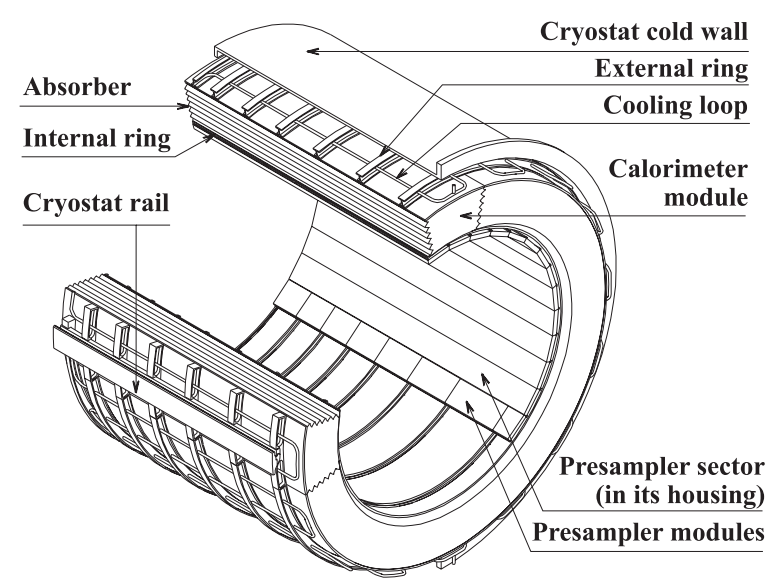

Figure 1. View of a barrel wheel. energy resolution it is necessary to correct for the energy lost upstream the calorimeter. For this purpose a separate presampler detector, providing a shower sampling in a thin LAr layer (11 $\mathrm{mm}$ in depth) was also developed and placed in front of the calorimeter [2] (fig. 1). Both detectors: calorimeter and presampler, are placed in the same cryostat filled with liquid argon. Actually the whole detector is made of two barrel wheels, centered around the z-axis (ATLAS beam axis). The length of each wheel is $3.2 \mathrm{~m}$ (covering the region $0 \leq|\eta| \leq 1.475$ ); the inner and outer diameters are about 2.8 and $4 \mathrm{~m}$, respectively.

\section{THE BARREL PRESAMPLER}

Sixty four sectors, each composed of 8 different types of modules, are needed for the whole detector (fig. 2). The modules are made of interleaved cathodes and anodes, glued between FR4 plates. Anodes are 3 conductive layered boards; a high voltage of $+2 \mathrm{kV}$ is applied to the outer layers and the signal is read out through capacitive coupling to the central anode layer. The required granularity $(\Delta \eta=0.025, \Delta \phi=0.0982)$ is obtained by ganging the appropriate number of anodes in the $\eta$ (or z) direction and by subdividing (i.e. etching) each anode into two compartments in the $\phi$-direction.

There are 2 assembly sites (one in Grenoble and 


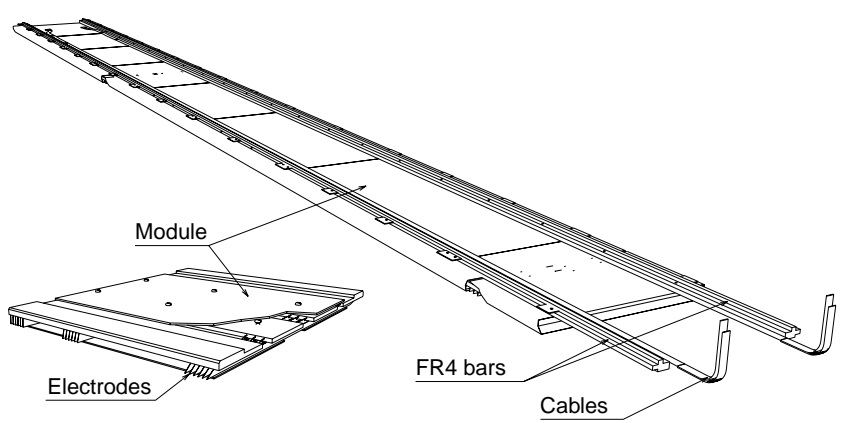

Figure 2. Perspective view of a presampler sector.

the other one in Stockholm), and each of them is equipped with a cold station. Each sector is checked on a test bench first at room temperature and then in liquid nitrogen. A high voltage test is performed and the response of each channel is studied (response to test pulses and noise measurement). All electrical connections are checked by connecting the HV inputs to a low voltage and low frequency sinusoidal signal.

By the end of October 2002, 38 sectors have been produced and tested, allowing the insertion of the presampler in the first calorimeter wheel (only 32 sectors are needed). This operation will be achieved in November 2002 when the calorimeter wheel will be put in its horizontal position. The last sector for the second wheel is expected at the end of May 2003.

\section{THE BARREL CALORIMETER}

Thirty two calorimeter modules (16 per wheel) are needed for the whole detector. Each module is about $47 \mathrm{~cm}$ in depth, and is composed of 64 absorbers and 64 electrodes with an accordion geometry in the $(\mathrm{R}, \phi)$ plane (see fig. 3 ), allowing a full azimuthal acceptance.

\subsection{Absorbers, electrodes and spacers}

The absorbers are made of lead (thickness $1.53 \mathrm{~mm}$ for $|\eta|<0.8,1.13 \mathrm{~mm}$ for $|\eta|>0.8)$ protected by two stainless steel sheets $(0.2 \mathrm{~mm}$ thick) glued on the lead and keeping the nominal thickness of the absorber to $2.2 \mathrm{~mm}$. When ar-

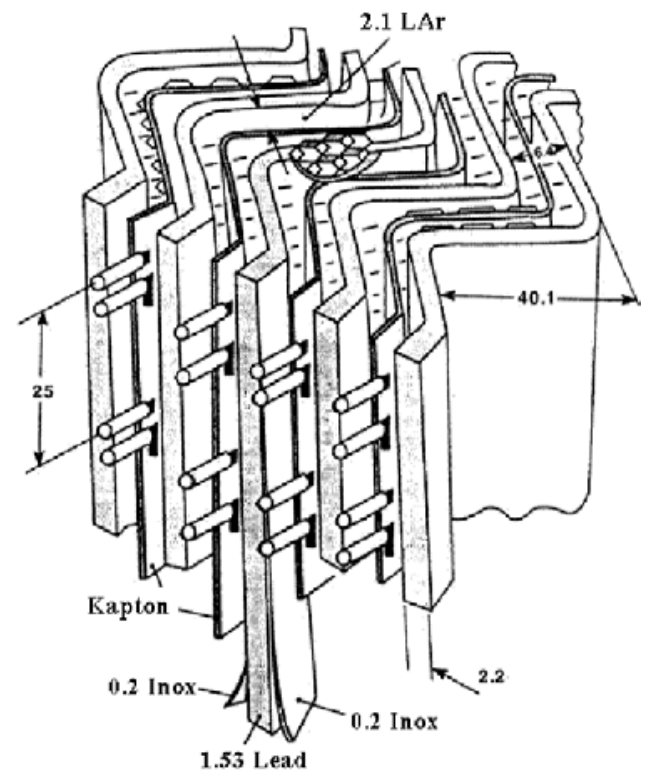

Figure 3. Sketch of the ATLAS liquid argon electromagnetic calrimeter accordion structure.

riving to the laboratory the thickness of the lead sheets is measured by means of an ultrasound technique. A sorting algorithm is used to match the lead plates in order to compensate for local non-uniformities. The expected contribution to the constant term is between 0.1 and $0.2 \%$. The end of production is expected in January 2003.

The electrodes are 3 copper layers insulated by two kapton layers. The 2 external copper planes are connected to the high voltage $(+2 \mathrm{kV})$ and the signal is read out through capacitive coupling to the central copper layer. Each gap between 2 absorbers is equipped with electrodes of two types: A $(|\eta|<0.8)$ and $\mathrm{B}(|\eta|>0.8)$. The geometry of the detection cells in $\eta$ is projective with respect to the collision point and is obtained by etched patterns. The HV protection resistors pads are silk screened components based on resistive ink. The production of the flat electrodes ended in July 2002 (including $10 \%$ spares). After bending, the resistive pads are mesured individually for each electrode on dedicated test benches. 


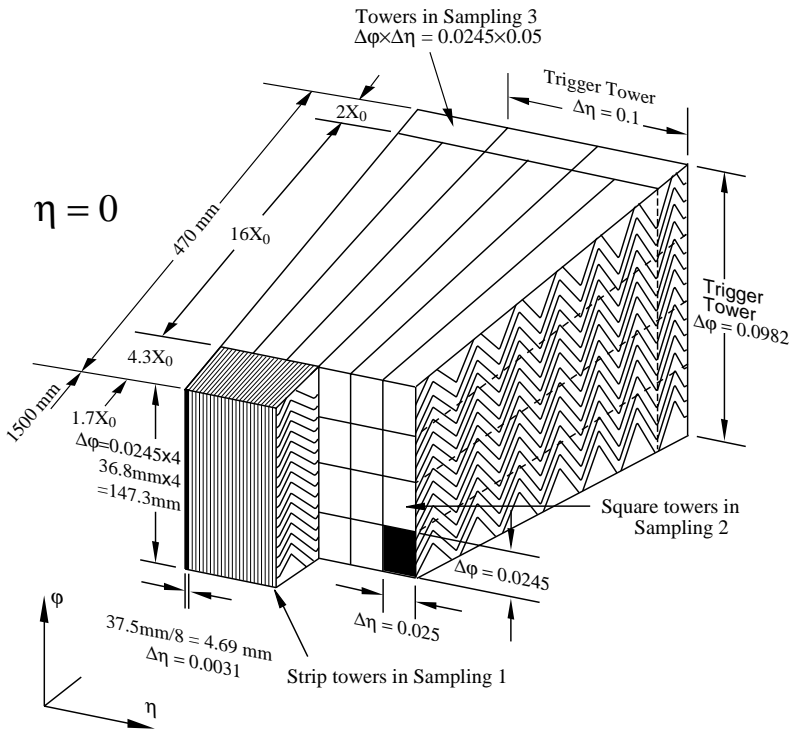

Figure 4. Sketch of the accordion structure of the EM calorimeter.

Damaged resistors can be repaired. The end of tests and equipments are expected in November 2002 for the B electrodes and February 2003 for the A electrodes.

The absorbers and electrodes are kept in place by means of honeycomb spacers. These spacers are tested at $3 \mathrm{kV}$ after cleaning and before stacking.

\subsection{Calorimeter granularity}

The granularity in $\phi$ is obtained by grouping together a proper number of electrodes (see fig. 4), and the granularity in $\eta$ is obtained by etching the readout electrodes. There are 3428 readout cells per module.

The detector is also segmented into 3 radial compartments:

- The strips, allowing a very accurate position measurement in $\eta$ and the $\gamma / \pi^{0}$ separation. It has a granularity of $\Delta \eta=0.0031$, $\Delta \phi=0.0982$.

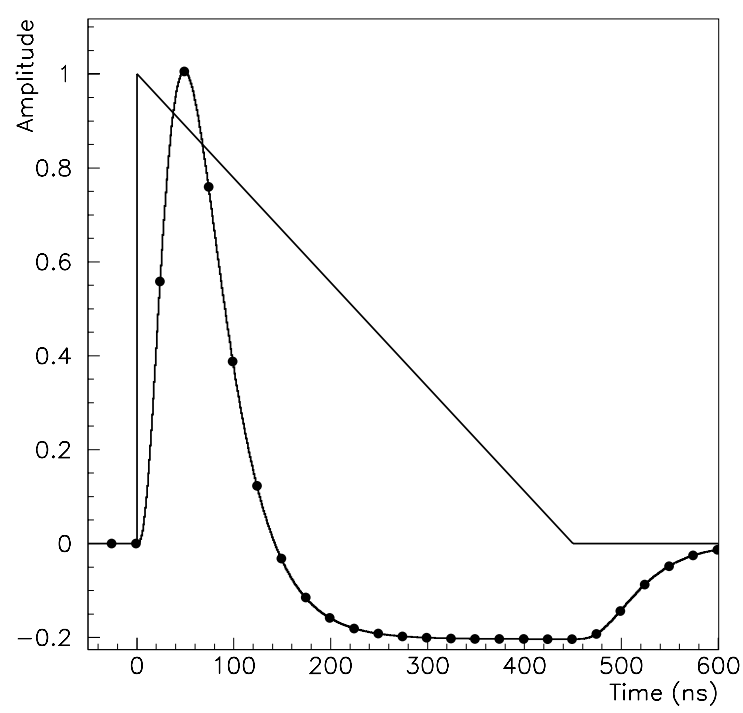

Figure 5. Signal shape in the detector (triangle) and after shaping (curve with dots).

- The middle compartment, where most of the energy is deposited. Its granularity is $\Delta \eta=0.025, \Delta \phi=0.0245$.

- The back compartment. It has a granularity of $\Delta \eta=0.050, \Delta \phi=0.0245$.

\subsection{Module assembly}

Three sites (CERN, Saclay and Annecy) are fully operational. The stacking is done on a rotating jig in a clean room. The first absorber is fixed in place with references in all axes (to about $0.1 \mathrm{~mm}$ ). A first spacer plane, the electrode plane, a second spacer plane and a new absorber are stacked. HV and continuity tests (i.e. injection of low frequency sinusoidal signal in the HV lines) are systematically performed for each gap before proceeding to the next one. It was also decided: 1) to add protective diodes on all modules in order to protect the very accurate calibration resistors $(0.1 \%)$ in case of short-circuit, 2$)$ to use spare HV channels to split faulty gaps. 


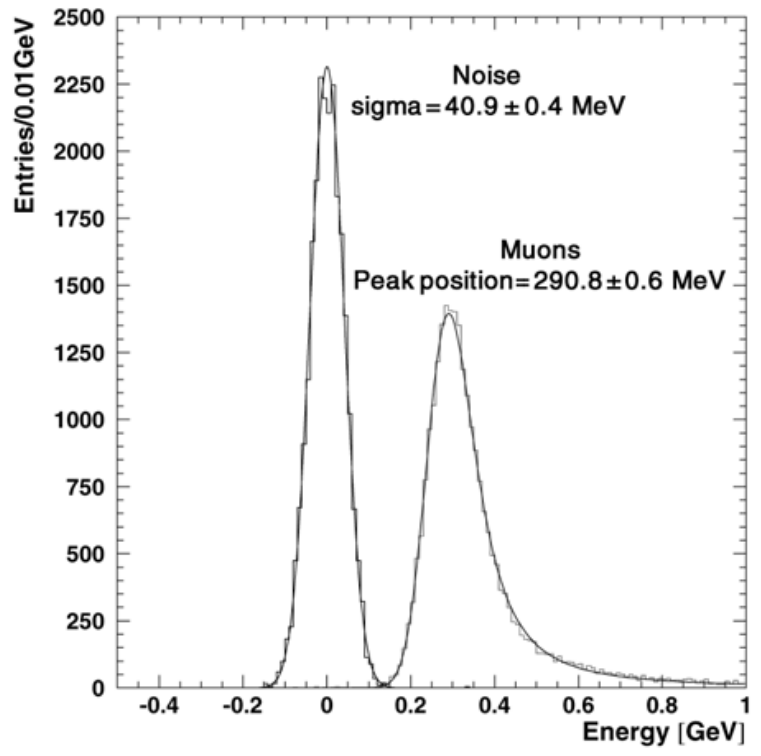

Figure 6. Muon response in the middle compartment and noise distribution.

\subsection{Barrel assembly}

At the end of October 2002, the first barrel calorimeter wheel (i.e. 16 modules) was finished assembling at CERN without meeting major difficulties. The assembly of the second wheel is foreseen in June 2003.

\section{TEST BEAM RESULTS}

The system: one calorimeter module + two presampler sectors has been tested several times on the $\mathrm{H} 8$ beam line of the CERN SPS.

Fig. 5 shows the signal shape produced in the detector cells (triangle), and after the CR-RC ${ }^{2}$ shaping (curve with dots). Only 5 samples on the positive lobe are recorded. The maximun amplitude of the peak $\left(A_{\max }\right)$ is deduced from an optimal filtering technique [3]:

$$
A_{\text {max }}=\sum_{i=1}^{5}\left(a_{i} . S_{i}\right) \quad A_{\text {max }} . \Delta t=\sum_{i=1}^{5}\left(b_{i} . S_{i}\right)
$$

where $S_{i}$ is the sampled signal at time $t_{i}, \Delta t$ is the deviation from assumed time, and the coefficients

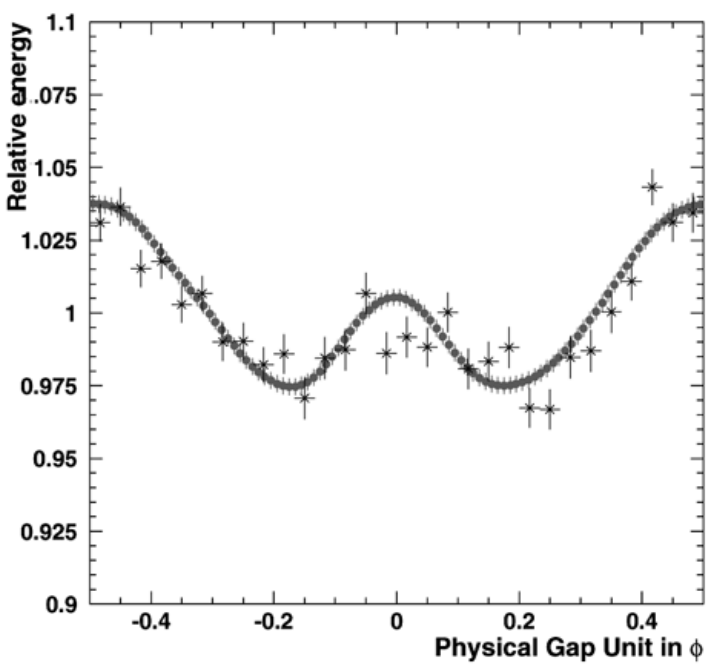

Figure 7. $\phi$ modulation obtained with muons (experimental points) and with a Monte Carlo simulation (solid curve).

$a_{i}$ and $b_{i}$ are computed in order to minimize the noise.

By using a reference cell, it was observed that the stability of the measured energy of a $250 \mathrm{GeV}$ electron beam was equal to $0.1 \%$ over 9 days.

\subsection{Response to muons}

Muon data were collected by exploiting the large $\mu$ contamination in the electron beams [4]. The signal was reconstructed using a $1 \times 2$ cluster in $\eta \times \phi$ in the middle sampling. The cell selection was made event by event, using the particle impact point extrapolated from beam chambers. The signal is plotted in fig. 6 and is well separated from the random (i.e. noise) distribution. The signal/noise ratio is equal to $7.11 \pm 0.07$.

Muons are minimum ionising particles and therefore provide cleaner or complementary information. For example fig. 7 shows the peak to peak amplitude of the $\phi$ modulation (about $6 \%$ ) due to the absorber and electrode structure. This modulation is 4 times larger than what can be observed with electrons. The solid curve in fig. 7 represents the results obtained with a Monte Carlo (Geant3) 


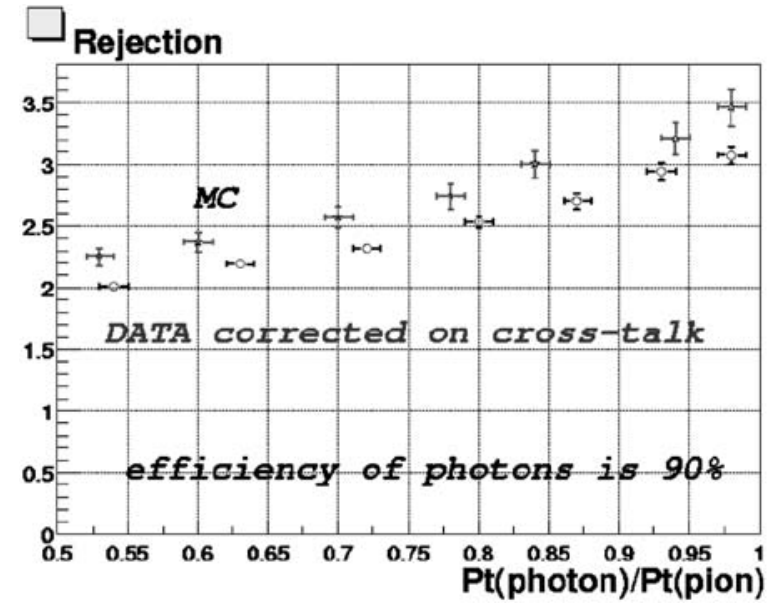

Figure 8. Rejection factor, see text.

simulation. In this case the map of the electrical field, in particular at the top of the folds, has been taken into account. One can see that the agreement is quite good.

\section{2. $\gamma / \pi^{0}$ separation}

The aim of this study is to reject the neutral pions in order to get a better identification of the Higgs decaying into 2 photons $(\mathrm{H} \rightarrow \gamma \gamma)$. Photon data were obtained from special runs where the electron beam was hitting a target in order to produce photons. Electrons were then deviated by a powerful magnet, in such a way that both the electron and the bremsstrahlung photon were detected simultaneously by the calorimeter. The next step consists in simulating the decay of the $\pi^{0}$ into 2 photons $\left(\pi^{0} \rightarrow \gamma \gamma\right)$ with a transverse momentum $\left(\mathrm{P}_{t}\left(\pi^{0}\right)\right)$ of $50 \mathrm{GeV} / \mathrm{c}$. Then the simulated photons are replaced by real ones with the correct kinematics. Neutral pions are separated from single photons by applying a shape analysis in the strip compartment. A rejection factor is defined as follows: $\mathrm{R}=(\gamma$ efficiency $) /(1-$ $\pi^{0}$ rejection rate). Fig. 8 shows our results. The average rejection factor for a $\gamma$ efficiency of $90 \%$ is $\langle\mathrm{R}\rangle=2.6 \pm 0.05$ after correcting for crosstalk. This factor can be compared with the prediction of a Monte Carlo simulation which includes the electronic noise and gives $<\mathrm{R}>=2.8 \pm 0.1$.

\section{CONCLUSIONS}

The construction of the barrel EM calorimeter and presampler is proceeding well. A big effort on the quality controls of the main components (absorbers, electrodes etc.) and on the validation tests of the modules or sectors has been made. The assembly of the first half barrel has already started: the first calorimeter wheel is already completed and the presampler sectors will be inserted in November 2002.

The test beam results show that the performance is within expectations, for example the signal to noise ratio for muons is equal to $7.11 \pm 0.07$ and a $\gamma / \pi^{0}$ rejection factor of 2.6 with a $\gamma$ efficiency of $90 \%$ has been measured.

\section{REFERENCES}

1. D.M. Gringrich et al. (RD3 Collaboration), Nucl. Instr. and Meth. A 364 (1995) 290.

2. M.L. Andrieux et al., Nucl. Instr. and Meth. A 479 (2002) 316.

3. W.E. Cleland and E.G. Stern, Nucl. Instr. and Meth. A 338 (1994) 467.

4. Performance of the ATLAS electromagnetic calorimeter barrel module 0; B. Aubert et al. (ATLAS Electromanetic Liquid Argon Calorimeter Group); to be published in Nucl. Instr. and Meth. A. 